\title{
Amorphous Antimony in Thin Films
}

\author{
Styrkas A. D.* \\ In Solid St. Phys RAS Chernogolovka Russia.
}

*Corresponding Author: Styrkas A. D. In Solid St. Phys RAS Chernogolovka Russia.

Annotation: The conditions for obtaining amorphous $S b$ films by electrode position from various solutions and vacuum evaporation are studied. It is shown that the explosion Sb is determined by the phase transformation into a stable phase, and not by impurities $C l$.

Keywords: Sb amorphous electrolytic vacuum explosiveness mechanism.

\section{Preface}

It is considered to be the existence of Sb 4 modifications [1]: 1) crystal $\alpha$ Sbhexagonal; 2) below $90^{\circ} \mathrm{C}$ yellow, blackening in light and heating with a transition to 3) black form; and 4) "explosive" $\beta$ $\mathrm{Sb}$. It is believed that $\beta \mathrm{Sb}$ is a solid chlorine solution in $\mathrm{Sb}$ and is formed by electrolysis of chloride solutions at high current density. This statement is found in many textbooks.

\section{EXPERIMENTAL PART}

Polarization curves i - $\varphi$ were removed galvanostatically in an inert gas atmosphere with stirring against a saturated cacalomel reference electrode. Cathode $-\mathrm{Pt}$ polished plate area $1 \mathrm{sm}^{2}$, all $\varphi$ related to normal hydrogen. Analysis - amperometric titration by [2]. The cathode space was separated from the anode from anodic porous partitions. The current output was determined by weighing the cathode. Total Q passing through the cell, electricity controlled copper Q-meter with a solution of Eitel. Reflectance R of films was measured on a GOI reflectometer with Se photocell, vacuum deposition was carried out at specified distances from the crucible with $\mathrm{Sb}$ heated by a spiral from $\mathrm{W}$ to substrates of transparent polished $\mathrm{SiO} 2$ for adhesion, the Color of the amorphous film treated with a weak solution of $\mathrm{SnCl}$ and its transition to a brilliant $\alpha \mathrm{Sb}$ Was noted visually. The electrical conductivity of the film during the transition from amorphous to crystalline state changed $\sim 500$ times.

It turns out that the so-called $\beta$-form of "explosive antimony" does not contain chlorine. This is proved by chemical analysis. The existence of metastable amorphous $\mathrm{Sb}$ passing to $\alpha \mathrm{Sb}$ under energy influences was found.

Analysis by [2] show that $\mathrm{Sb}$ obtained by electrode position from a solution of $\mathrm{SbCl} 3$ in $\mathrm{HCl}$ acid in strongly acidic medium gave the absolute absence of $\mathrm{Cl}$ in the sediments. The explosion of the sediment is not due to the presence of $\mathrm{Cl}$, but because it has an amorphous metastable structure. Its amorphous proven by X-raying. The debaegram has a clean background with a negligible hint of $\mathrm{Sb}$. Exploding gives from amorphous Sbthe $\alpha \mathrm{Sb}$ dust during the exothermic transition, as the explosion tetragonal, InSb obtained at low $\mathrm{T}$ and high P.The abundance of allotropic phases complicates the electrode position of Sbfromthe $\mathrm{SbCl} 3$ solutions, explosive Sbgrows at the cathode, which was explained by the presence of $\mathrm{Cl}$ impurities. The collected fine dust $\mathrm{Sb}$ also does not contain $\mathrm{Cl}$. Additives to surfactant solution to increase (-) the potential of isolation such as diphenylamine, thymol, $\beta$ naphthol release potential $\varphi$ from $+0.2 \mathrm{~V}$ is shifted to $-0.85 \mathrm{~V}$, giving compact shiny precipitates contaminated with inclusions of organic impurities. This precipitation is also amorphous, but not explosive and do not contain $\mathrm{Cl}$, and whenSb heated, blackened from charring of organic impurities captured Strip out impurities slows down the process of transition into the stable phase, and the explosion does not happen. Also in vacuum at the spraying to cold $\mathrm{SiO}_{2}$, remote from the crucible, settled brown oily spot, but it soon as quickly as the explosion instantly turned into a brilliant crystalline film Sb too, but without the spread of dust particles. The growth of the phase provided the process with energy, the analysis gave $100 \% \mathrm{Sb}$, proving that it was a transition from the amorphous 
phase to a stable one. With rapid condensation on cold $\mathrm{SiO}_{2}$ and a small thickness of the nano-sized film, the metastable phase can exist, but passes into the crystal stable $\alpha$-phase under energy influences. At a close nearby distance from the evaporator, this phenomenon can't be observed due to the heating of the evaporator by radiation. A similar phenomenon is observed in the amorphous $\mathrm{Sb}$ obtained by electrolysis in the presence of surfactive substances Shiny amorphous cathode films on $\mathrm{Pt}$ and $\mathrm{Cu}$ foil explode with sound when heated, but without sputtering due to organic impurities inhibiting the atomization. The light $\mathrm{Sb}$ from the sulfuric acid baths at the bend makes a sound similar to cry at the bend Sn. Covalent brittle crystallites give rise to a cry when friction. Bending of amorphous cathode sediments obtained in the presence of surfactant does not give cry. There is no sound in precipitation from sulphate solutions in which $\mathrm{Sb}_{2}\left(\mathrm{SO}_{4}\right)_{3}$ is slightly soluble. In a solution of $1.54 \mathrm{~g} / \mathrm{Sb}_{2}\left(\mathrm{SO}_{4}\right)_{3}$ in $10 \mathrm{n} \mathrm{H}_{2} \mathrm{SO}_{4}$ at a current density i below $0.5 \mathrm{~mA} / \mathrm{sm}^{2}$ crystal light $\mathrm{Sb}$ grows. At $\mathrm{i}=0.2 \mathrm{~mA} / \mathrm{sm}^{2}$ polishing gives a reflection coefficient $\mathrm{R}$ of almost $100 \%$, as from vacuum films. With growth i R of the depositions with steeply falls. At i from $0.6, ; 0.7 ;$ and $1 ; 2 \mathrm{~mA} / \mathrm{cm}^{2} \mathrm{R}$ obtained respectively $40 \% ; 9 \% ; 2 \%$, at higher i grows black loose sediment $\mathrm{Sb}$ with $\mathrm{R}=0$ at particles crystalline and fine. The current output drops, but smoothly (from $100 \%$ at 0.2 . to $12 \%$ at $10 \mathrm{~mA} / \mathrm{sm}^{2}$ ) At i above id not only do powders and dendrites grow, the current output drops too. Decreases the release of the $\mathrm{Sb}$ due to its restoration to $\mathrm{SbH}_{3}$. (Fig 1) the Formation of $\mathrm{SbH}_{3}$.s also proven analytically.

Similarly, when large oversaturated and at high speed formation of nanoparticles of metastable phase, even non-existent in the nature of the W-phase CdTe describes the real preparation of the $\mathrm{W}$ phase, but not S CdTe [3], that allowed to investigate a number of optical and other interesting properties of this unknown structure. Nanoparticles of sizes less than $1 \mathrm{~nm}$ of phase unknown in the nature, naturally, metastable, but capable to exist. Diamond-like structure $\mathrm{S}$ of semiconductor CdTe has very close lattice parameters to its counterpart of semiconductor $\alpha \mathrm{Sn}$, so it can infect the $\beta \mathrm{Sn}$.All connections A3Б5 can have both structure and S, and W, but only for CdTe structure is missing in nature! Cubic phase of CdTe can infect $\beta \mathrm{Sn}$ to $\alpha \mathrm{Sn}$, but tetragonal phase W CdTe can't infects. So them easy distinguishing! Later with exposure and energy assistance, this metastable phase passes into a cubic stable S phase of CdTe and infection occurs, proving the description of the mechanism of formation of even non-existent in nature W CdTe Phase W and its the subsequent transition to a stable phase $\mathrm{S}$, infecting tin. It is possible to create something that was not in nature, we must to do need conditions. So it is possible to create and metastable non-existing tetragonal In Sb by obtaining at low $\mathrm{T}$ and high $\mathrm{P}$. Introduced under normal conditions, it explodes into the dust of the $\mathrm{S}$ structure. (InSb is the isoelectronic and structural analogue of the semiconductor $\alpha \mathrm{Sn}$ ) the Transition from diamond-like covalent $\alpha \mathrm{Sn}$ to tetragonal metal $\beta \mathrm{Sn}$, stable phase similarly, only in the opposite structural direction, converts $\mathrm{Sn}$ into powder from mechanical stresses with a change in the volume of the structure by $26.6 \%$ with similar mechanical breakdowns. So is method of receiving of pure Sn powder bymany times repetition of $\beta \mathrm{Sn} \rightarrow \alpha \mathrm{Sn}$ and $\alpha \mathrm{Sn} \rightarrow \beta \mathrm{Sn}$, without explode.

The abundance of allotropic phases in Sb complicates electrode position. At large super saturation in solutions $\mathrm{SbCl} 3$ grows at the cathode explosive $\mathrm{Sb}$,that was explained by the presence of impurities $\mathrm{Cl}$. And the collected fine dust $\mathrm{Sb}$ doesn't contain $\mathrm{Cl}$. Additives to the solution of surfactants to increase the - $\varphi$ of the potential of deposition by diphenylamine, thymol, $\beta$-naphthol from $+0.2 \mathrm{~V}$ shift to $-0,85 \mathrm{~V}$, giving a compact shiny precipitates contaminated with inclusions of organic impurities. Precipitation is also amorphous, but not explosive and do not contain $\mathrm{Cl}$, and when heated, blackened from charring of organic impurities captured Strip out impurities slows down the process of transition into the stable phase, so the explosion does not happen.

When spraying in vacuum on cold $\mathrm{SiO}_{2}$, removed from the crucible, settled brown oily spot, but it soon as quickly as the explosion instantly turned into a brilliant crystalline film $\mathrm{Sb}$, but without the spread of particles. The phase growth provided the process with energy, the Analysis gave $100 \% \mathrm{Sb}$, proving that it is also transition from amorphous phase to stable. At rapid condensation on cold $\mathrm{SiO} 2$ and at small thickness of the nano-sized film, the metastable phase can exist, but passes into the crystal stable $\alpha$-phase under the energy effects of the phase growth. At a close distance from the evaporator, this phenomenon cannot be observed due to the heating of the evaporator by radiation. A similar phenomenon is observed in the amorphous $\mathrm{Sb}$ obtained by electrolysis in the presence of surfactants, Shiny amorphous cathode films on $\mathrm{Pt}$ and $\mathrm{Cu}$ foil explode with sound when heated, but without spraying due to organic impurities inhibiting the spray. The light $\mathrm{Sb}$ from the sulfuric acid baths at the bend makes a sound similar to the cry at the bend Sn. Covalent brittle crystallites give rise 
to a cry at the friction. Bendingo famorphous cathode sediments obtained in the presence of surfactant do not give cry. There is no sound in precipitation from sulphate solutions in which $\mathrm{Sb}_{2}\left(\mathrm{SO}_{4}\right)_{3}$ is slightly soluble. In a solution of $1.54 \mathrm{~g} / \mathrm{l}$ sulphate $\mathrm{Sb}$ in $10 \mathrm{n}$ acid at a current density i below 0.5 $\mathrm{mA} / \mathrm{sm}^{2}$, crystal light Sb grows. At $\mathrm{i}=0.2 \mathrm{~mA} / \mathrm{sm}^{2}$ polishing gives a reflection coefficient $\mathrm{R}$ of almost $100 \%$, as from vacuum films. With growth i R with steeply fall. At $\mathrm{i}=0.6, ; 0.7$; and $1 ; 2 \mathrm{~mA} / \mathrm{sm}^{2} \mathrm{R}$, respectively, obtained 40\%; 9\%;2\%, atihigher $\mathrm{Sb}$ grows black loose sediment with $\mathrm{R}=0$ of small crystalline particles.

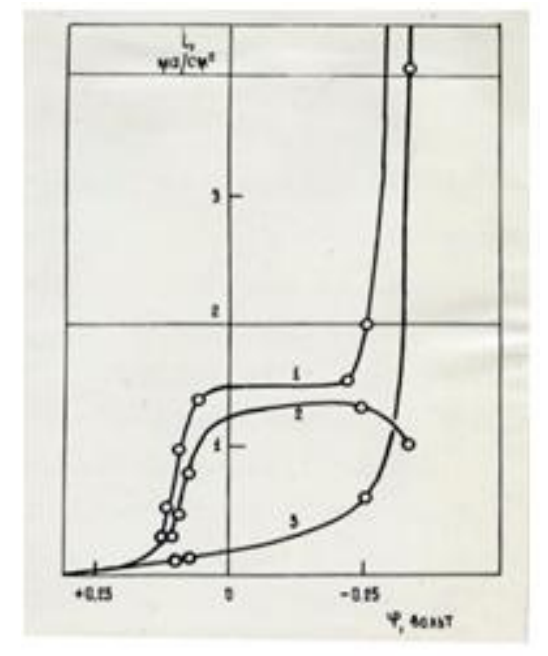

Fig1. Common cathodic curves (1); and partial (2): the deposition of $\mathrm{Sb}$ decay shows the formation of $\mathrm{SbH}_{3}$ at $\varphi$ value more than -0.15; and (3) the desorption of $\mathrm{H}_{2}$.

The current output drops, but smoothly (from $100 \%$ at 0.2 . to $12 \%$ at $10 \mathrm{~mA} / \mathrm{sm}^{2}$ ) Ati above id not only do powder and dendrites grow, the current output drops. Decreases the release of the Sb due to its restoration to $\mathrm{SbH}_{3}$. (Fig1.) the Formation of $\mathrm{SbH}_{3}$ is proved analytically.

\section{SUMMARY}

Amorphous films were obtained by electrode position and vacuum deposition of antimony. It is proved that there is no previously considered $\beta$ - phase of antimony, and the so-called explosive antimony, allegedly exploding from the content of chlorine in it is a metastable amorphous phase capable of existing in nano particle sizes and passing into a stable $\alpha$-phase with energy reins and growth of particle sizes, like other metastable systems.

\section{REFERENCES}

[1] Remy,H. Course of inorganic chemistry IL., M., 1963, vol. 1. p.920

[2] Songina O. A. Amperometric titration in the analysis of mineral raw materials M., Gosgeoltekhizdat, 1957, p.304

[3] A.D. Styrkas. A. D. CdTe Nanoparticles and Clusters. //Inorganic Materials. 2008. v. 44, No 5, pp. 470-474.

Citation: Styrkas A. D., (2019). “Amorphous Antimony in Thin Films”. International Journal of Advanced Research in Chemical Science (IJARCS), 6(3), pp.22-24. DOI:http://dx.doi.org/ 10.20431/ 2349-0403.0603003.

Copyright: (C) 2019 Authors. This is an open-access article distributed under the terms of the Creative Commons Attribution License, which permits unrestricted use, distribution, and reproduction in any medium, provided the original author and source are credited. 